\title{
Multi-party Finite Computations *
}

\author{
Tomasz Jurdziński*, Mirosław Kutyłowski*†, and Krzysztof Loryś* \\ * Institute of Computer Science, Wrocław University \\ $\dagger$ Department of Mathematics and Computer Science, Poznań University
}

\begin{abstract}
We consider systems consisting of a finite number of finite automata which communicate by sending messages. We consider number of messages necessary to recognize a language as a complexity measure of the language. We feel that these considerations give a new insight into computational complexity of problems computed by read-only devices in multiprocessor systems. Our considerations are related to multi-party communication complexity, but we make a realistic assumption that each party has a limited memory.

We show a number of hierarchy results for this complexity measure: for each constant $k$ there is a language, which may be recognized with $k+1$ messages and cannot be recognized with $k-1$ messages. We give an example of a language that requires $\Theta(\log \log n)$ messages and claim that $\Omega(\log \log (n))$ messages are necessary, if a language requires more than a constant number of messages. We present a language that requires $\Theta(n)$ messages. For a large family of functions $f$, $f(n)=\omega(\log \log n), f(n)=o(n)$, we prove that there is a language which requires $\Theta(f(n))$ messages. Finally, we present functions that require $\omega(n)$ messages.
\end{abstract}

\section{Introduction}

Today, communication capacity is one of the most important bottlenecks of computer systems. For this reason, there is a growing interest in communication complexity of problems. From a practical point of view, corresponding complexity measures are often more important than traditional measures such as time and space complexities.

Communication complexity has been introduced as two-party communication complexity. For this measure, an input is divided between two processing units that compute together the output [8]. Communication complexity is defined as the number of message bits exchanged between the processing units. Despite many efforts, the number of results obtained for this measure is relatively low, proving that the field belongs to difficult parts of theoretical computer science. The results that have been obtained so far are often very involved (see for instance [8]).

Development of distributed computing systems increased interest in multi-party communication complexity. Also, it became clear that interprocessor communication is a crucial limitation for parallel systems and that good parallel algorithms must be communication efficient. Two-party communication complexity has been generalized to some extent to multi-party communication complexity. However, these results often assume specific architecture (see for instance [4]).

The approach discussed above is based on the assumption that during a two-party computation communication is the most crucial resource and the size limitation of the

\footnotetext{
^ partially supported by Komitet Badań Naukowych, grant 8 T11C 03215
} 
internal memory can be neglected in order to keep the picture clear. However, often this is not a good description of a real situation. For instance, on a multiprocessor system, computational capacity of each processing unit might be severely limited.

In this paper we consider a situation when each processing unit has a finite memory, that is, it is a finite automaton. (Our results can be easily extended to the situation, when each of the processing units has some small internal memory.) The input is not divided between the processing units. It is stored in a read-only memory and is available for each processing unit. We consider the processing units to be automata with read heads, but their sequential access to input data has no direct influence on the amount of communication between the processing units. Thus, our considerations are relevant for large problems with input data stored in a read-only shared memory, computed by systems of many processing units of limited internal memory communicating by messages.

Research on coordination means of systems of finite automata have been initiated in $[3,6]$. Somewhat related are also the papers on the trade-off between computation time and space of multihead automata [5,7]. However, these results are based on the fact that in order to examine distant parts of the input the heads must spend quite a long time to get there. So they are not relevant for the size of communication between automata.

A significant step in understanding communication complexity was to apply ideas of Borodin, Cook [2] and Yesha [11] to two-party computation. A trade-off for communication time and space has been shown for problems such as matrix multiplication $[1,9]$. Here, communication time relates to the number of bits exchanged and space denotes logarithm of the number of internal states. In the strongest version, the computing model assumed is very general (i.e. branching programs). The results mentioned have some impact on what we can say about systems of finite automata, since their computations may be simulated by two "agents" communicating through messages.

Model We consider systems consisting of a finite number of finite automata working independently, except that they may communicate by broadcasting messages. (Since we consider systems of finitely many automata, this is the same, up to a constant factor, as messages delivered in a point-to-point fashion.) Since each automaton has only finitely many internal states, the number of different messages is finite, as well. Transition function of a single automaton depends on its internal state, an input symbol seen and all messages received from the other automata at this moment. The messages are delivered instantly. The automata work synchronously. More formally, we define such systems as follows:

Definition 1. $M=\left(A_{1}, A_{2}, \ldots, A_{l}\right)$ is an l-automata system, if the following conditions hold. There is a finite set $Q$ such that every internal state of any $A_{i}$ is in $Q$. Each automaton has a single read-only head. Let automaton $A_{i}$ be in state $q$ and let a be the input symbol seen by $A_{i}$. Then the next step is executed by $A_{i}$ as follows:

(1) $A_{i}$ broadcasts a message $m=\mu_{i}(q, a)$ to other automata. If $\mu_{i}(q, a)=$ nil, then no message is sent by $A_{i}$.

(2) $A_{i}$ changes its internal state to $q^{\prime}$ and moves its read head $r$ positions to the right $(r \in\{0,1,-1\})$, where $q^{\prime}$ and $r$ are given by transition function $\delta_{i}$ :

$$
\left(q^{\prime}, r\right)=\delta_{i}\left(q, a, m_{1}, \ldots, m_{i-1}, m_{i+1}, \ldots, m_{l}\right)
$$

where $m_{j}$ denotes the message sent by automaton $A_{j}$ during the first phase of the step (if no message is sent by $A_{j}$, then $\left.m_{j}=n i l\right)$. 
We say that an l-automata system accepts a language $L$, when a fixed automaton (for instance the first one) enters an accepting state on input $x$ if and only if $x \in L$.

Complexity classes We define $\operatorname{MESSAGE}_{l}(f(n))$ as the class of all languages that may be recognized by an $l$-automata system where at most $f(n)$ messages are sent for every input word of length $n$. By $\operatorname{MESSAGE}(f(n))$ we denote $\bigcup_{l \in \mathbb{N}} \operatorname{MESSAGE}_{l}(f(n))$. Note that even $\operatorname{MESSAGE}_{2}(1)$ contains non-regular languages. Indeed, $\left\{a^{m} b^{m} \mid m \in \mathbb{N}\right\} \in$ $\operatorname{MESSAGE}_{2}(1)$. Similarly, $\left\{a^{m} b^{m} c^{m} \mid m \in \mathbb{N}\right\} \in \operatorname{MESSAGE}_{3}(1)$ but is not context-free.

New results We prove a number of hierarchy results for MESSAGE complexity measure. The first one is the hierarchy result for a constant number of messages:

Theorem 1. $\operatorname{MESSAGE}_{l}(k-1) \subsetneq \operatorname{MESSAGE}_{l}(k+1)$, for all $k \geq 1$ and $l>1$.

We present an example of a language that requires a double-logarithmic number of messages:

Theorem 2. For $a, b \in \mathbb{N}$, let

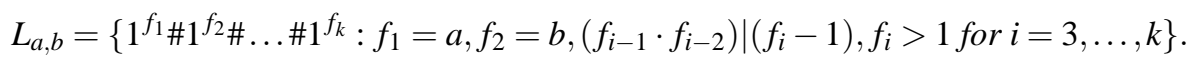

If $a, b>1$ are prime, then $L_{a, b} \in \operatorname{MESSAGE}_{2}(O(\log \log n))$ and $L_{a, b} \notin \operatorname{MESSAGE}_{l}(f(n))$ for any $f(n)=o(\log \log n)$ and $l \in \mathbb{N}$.

The following example shows that there are languages requiring $\Omega(n)$ messages:

Theorem 3. Let $L_{x x}=\left\{x \# x: x \in\{0,1\}^{*}\right\}$. Then $L_{x x} \notin \operatorname{MESSAGE}_{l}((f(n)))$, for any $l \in \mathbb{N}$ and $f(n)=o(n)$. (While $L_{x x} \in \operatorname{MESSAGE}_{2}(n)$.)

We already know that there are languages requiring $\Theta(\log \log (n))$ and $\Theta(n)$ messages, respectively. It is interesting to see that there is a dense hierarchy between these bounds.

Theorem 4. There exists a constant $c>0$ such that for any Turing machine timeconstructible function $f(m), f(m)=o\left(2^{2^{c m}}\right)$, there exists a language L which can be recognized by a 2-automata system $M$ which uses $O\left(f^{2}(\log \log n)\right)$ messages and cannot be recognized by a multi-automata system (with an arbitrary number of automata) which uses $o\left(f^{2}(\log \log n)\right)$ messages.

In particular, for simple functions $f$ such as $n^{c}$ for $c<1$, and $\log ^{c} n$ for $c \in \mathbb{N}$ we may obtain a hierarchy result. Finally, we give examples when a superlinear number of messages is necessary:

Theorem 5. Computing product over $\mathbb{F}_{2}$ of square matrices with $n$ elements (giving the output through a one-way output tape) requires $\Omega\left(n^{3 / 2} / \beta(n)\right)$ messages, for any number of automata in the system and every $\beta(n)=\omega\left(\log ^{2}(n)\right)$. (By a standard algorithm $O\left(n^{3 / 2}\right)$ messages are sufficient.) 
Theorem 5 can be derived from the results on two-party communication complexity [1] through simulating a system of finite automata in a two-party communication model (see the forthcoming full version of the paper). Here we give a relatively simple and direct proof based on Kolmogorov complexity. Also, we may derive some hierarchy results for superlinear number of messages using results known for the class LOGSPACE. However, the last technique does not provide a tight hierarchy. A variant of Theorem 5 gives explicit examples of functions for bounds between $n$ and $n^{3 / 2}$.

We assume that the reader is familiar with Kolmogorov complexity concept ([10]), which is the most important tool in crucial moments of our proofs.

Organization of the paper In this extended abstract we present sketches of the proofs of Theorems 1 (Section 2), 2 and 4 (Section 3), and 5 (Section 4). The details and the omitted proofs will be included in the full version of the paper.

\section{Hierarchy for a constant number of messages}

We prove Theorem 1 by showing that the following languages separate the classes $\operatorname{MESSAGE}_{l}(k-1)$ and $\operatorname{MESSAGE}_{l}(k+1)$ :

$$
L_{k}^{l}=\left\{x \# y_{1} \# y_{2} \# \ldots \# y_{k} \mid \forall i \quad D\left(x, y_{i}\right)\right\}
$$

where $D\left(x, y_{i}\right)$ holds if the characters at positions $|x|, 2|x|, \ldots,(l-1)|x|$ in $y_{i}$ are ones.

First let us see that an $l$-automata system may recognize $L_{k}^{l}$ using $k+1$ messages. First, all automata work in a cycle of length $l$. During this cycle automaton $A_{i}$ makes exactly $i$ moves to the right. This is continued until $A_{1}$ reaches the endmarker terminating $x$. Till this moment, $A_{i}$ has moved $(i-1) \cdot|x|$ positions in $y_{1}$. Then the automata check if there are ones on positions $|x|, 2|x|, \ldots,(l-1)|x|$ in $y_{1}$. In order to check the form of $y_{2}$ all automata move their heads at the same speed to the right, and $A_{1}$ sends a message once it reaches the symbol \# marking the beginning of $y_{2}$. The computation continues this way until all $y_{i}$ are checked. Finally, $A_{2}, \ldots, A_{l}$ send messages saying if all tests were positive. This is organized so that only one message is sent: at step $i$ of the final phase, automaton $A_{i+1}$ sends its message if anything was wrong and the whole system halts in a rejecting state. If the characters seen by $A_{i+1}$ witness that the input is in $L_{k}^{l}$, then $A_{i+1}$ sends no message.

We argue that $L_{k}^{l} \notin \operatorname{MESSAGE}_{l}(k-1)$. Intuitively any two-way deterministic finite automaton cannot "remember" the symbol of $y_{i}$ on position $|x|$ at configuration in which the position of the automaton on $y_{i}$ is far from position $|x|$. Moreover, we show that for appropriate inputs the messages are sent only in configurations in which one of the automata is "near" symbol \# or an endmarker. It follows that using $l$ automata and sending $k-1$ messages we can check only $(k-1)(l-1)$ positions. But there are $k(l-1)$ positions to be checked. Below we sketch a proof based on this intuition.

Lemma 1. Let $A$ be a 2-way finite automaton and let $q$ be the number of states of $A$. Then there is a word $b=b_{L} b_{R}$ of length at most $2 q^{2}$ such that:

- if the head of A enters $b$ from the left, then it either

- leaves $b$ on the left side without reaching $b_{R}$, or

- loops inside $b_{L}$, or 
- leaves $b$ on the right side and for no $x \in\{0,1\}^{*}$, the head of A ever leaves $b$ to the left side while working inside $b x$.

- if the head enters $b$ from the right, then analogous properties hold.

Proof. A simple induction. We commence by considering the state $q_{1} \in Q$. If there is any word $v$ that causes $A$ to loop inside $v$ while entered $v$ from the left side in state $q_{1}$, then we put $b^{(1)}=v$. Otherwise, if there is any word $w$ that causes $A$ to leave $w$ to the left, when entered $w$ from the left in state $q_{1}$, then we put $b^{(1)}=w$ for some such a word $w$. Otherwise $b^{(1)}$ is an empty word. Then we consider $q_{2} \in Q$. If there is a word $v^{\prime}$ such that $A$ loops inside $b^{(0)} v^{\prime}$ if entered it from the left in state $q_{2}$, then we put $b^{(2)}=b^{(1)} v^{\prime}$. Otherwise, if there is $w^{\prime}$ such that $A$ leaves $b^{(1)} w^{\prime}$ to the left, after some computation within $b^{(1)} w^{\prime}$ started in state $q_{2}$ at the left side, then we put $b^{(2)}=b^{(1)} w^{\prime}$. Otherwise $b^{(2)}=b^{(1)}$. We proceed this way until we consider all states of $A$. We put $b_{L}=b^{(q)}$ (where $q=|Q|$ ). By reversing directions we construct $b_{R}$.

Let $b$ be a word given by Lemma 1 for an automaton $A$. We consider inputs of a special form consisting of bits separated by word $b$ : if $z=z_{1} z_{2} \ldots z_{n}, z_{i} \in\{0,1\}$ for $i \leq n$, then $b_{z}=b z_{1} b z_{2} b z_{3} \ldots b z_{n} b$. The purpose of these words $b$ is to enforce a quasi one-way work of $A$ on $z$. By Lemma 1 , we see that if the head of automaton $A$ enters a word $b_{z}$ from the left (right) and reaches $z_{1}\left(z_{n}\right)$, then it leaves $b_{z}$ to the right (left) after some number of steps. Indeed, since the head comes through $b$, it cannot loop inside any word starting with $b$ or go back through $b$ in the opposite direction as started.

Our goal now is to show that a finite automaton cannot recognize what symbol stands in the middle of a word, even if we inspect the computation time. In fact, this is not true at some specific situations. We prove our claim for inputs $b_{z}$, where $z$ is random in the sense of Kolomogorov complexity. By clock characterization of word $z$ we mean the set of tuples $\left(q_{1}, p, q_{2}, t\right)$ such that if the head of $A$ enters $b_{z}$ in the state $q_{1}$ on side $p \in\{$ Left, Right $\}$ of $b_{z}$, and reaches $z_{1}$ for $p=$ Left or $z_{n}$ for $p=$ Right, then the head leaves $b_{z}$ in the state $q_{2}$ on the other side of $b_{z}$ after $t$ steps. Thus, clock characterization gives all information provided by the outcome of the computation on $b_{z}$ including such "side channel" information as computation time.

Lemma 2. (Confusion Lemma) Let A be a two-way finite automaton and $b$ be the word given by Lemma 1. Let $n$ be a sufficiently large odd number, $z=z_{1} z_{2} \ldots z_{n}, z_{i} \in\{0,1\}$ for $i \leq n, K(z) \geq n-c \log n$ for some constant $c$, and $z_{n / 2}=1$. Then there is a word $z^{\prime} \neq z$ such that $z_{n / 2}^{\prime}=0$ and the clock characterizations of $z$ and $z^{\prime}$ are the same.

Proof. (sketch) Assume for simplicity that the clock characterization of $z$ consists of a single element $\left(q^{\prime}\right.$, Left $\left., q^{\prime \prime}, t\right)$. Consider a computation of $A$ starting in the state $q^{\prime}$ at the leftmost symbol of $b_{z}$. For $i \leq n$, let $q_{i}$ be the state of $A$ at the moment when the head reaches $z_{i}$ for the first time. (So, by definition of $b$, the head cannot return to $z_{i-1}$ anymore after this moment). We say that subsequence $q_{j}, q_{j+1}, \ldots, q_{j+h}$ is a cycle if $q_{j}=q_{j+h}$ and the states $q_{j}, q_{j+1}, \ldots, q_{j+h-1}$ are different. Obviously, every subsequence of $q_{1}, \ldots, q_{n}$ of length $q$ contains a cycle. Let $f$ be the cycle which occurs most frequently in $q_{n / 2}, \ldots, q_{n}$. So, it occurs $\Omega(n)$ times. There exists at least one occurrence of the first state of $f$ in $q_{1}, q_{2}, \ldots, q_{n / 4}$, say at position $j$. Indeed, otherwise $z$ would be compressible, i.e. $K(z)<n-c \log n$. Let $Z_{i}$ be the word obtained from $z$ by moving the subwords of $z$ corresponding to the first $i$ cycles $f$ from the second half of $z$ to position $j$. In this way, we obtain $\Omega(n)$ words $Z_{i}$ with the same clock characterization as $z$. 
If the number of bits moved left to obtain $Z_{i}$ from $z$ is smaller than $n / 4$, then the middle bit in $Z_{i}$ is some bit from the first half of $z$. Note that this situation occurs for $\Omega(n)$ cases of $i$. The lemma holds if one of those bits is a zero. Otherwise, $z$ contains ones on positions $n / 2-i|f|$ for $i=1,2, \ldots, s$, where $s=\Omega(n)$. This means, however, that we could compress $z$ by $\Omega(n)$ bits, a contradiction.

The proof easily generalizes to clock characterizations containing more, say $r$, elements. It suffices to consider sequences of $r$-tuples of states.

Lemma 3. (Border Lemma) Let $M$ be a multi-automata system that uses at most $c=$ $O(1)$ messages for every input of the form $\{0,1, \#\}^{*}$. Then there is $b \in\{0,1\}^{*}$ and a constant $d \in \mathbb{N}$ such that

(a) Word b satisfies the claim of Lemma 1 for each automaton of $M$.

(b) Assume that an automaton of $M$ sends a message while working on an input word $b_{z_{1}} \# b_{z_{2}} \# \ldots \# b_{z_{m}}, m \in \mathbb{N}, z_{i} \in\{0,1\}^{*}$ for $i \leq m$. Then at this moment some of the heads of $M$ occurs at distance at most $d \cdot c \cdot|b|$ from a symbol \# or an endmarker.

Proof. First we generalize the proof of Lemma 1 . The first change is that we consider all automata of $M$ in the inductive construction of $b_{L}$ and $b_{R}$. The old construction applies provided that each state $q \in Q$ is replaced by copies $q^{(1)}, \ldots, q^{(l)}$, where $q^{(i)}$ corresponds to automaton $A_{i}$ of $M$. At the construction step for $q_{j}^{(i)}$, we consider automaton $A_{i}$. This establishes part (a).

For part (b) we incorporate one more condition into definition of $b$. Suppose that we have to consider state $q$ corresponding to automaton $A_{j}$ and that we have already constructed a word $b^{\prime}$. We first check if there is a word $u$ such that $A_{j}$ sends a message while working on $b^{\prime} u$, if entered from the left in state $q$ and no message is received in the meantime from the other automata of $M$. If such a word $u$ exist, then we replace $b^{\prime}$ through $b^{\prime} u$. Intuitively speaking, if there is a trigger causing $A_{j}$ to send a message, then such a trigger is built in $b$.

Now consider a computation of $M$ on an input of the form $b_{z_{1}} \# b_{z_{2}} \# \ldots \# b_{z_{m}}$. We distinguish periods, called later internal periods, when no automaton sees an endmarker or an \#. Consider an internal period $\tau$. Let $A_{p}$ denote an automaton that has left an endmarker or an \# at the beginning of period $\tau$. Let $\rho$ denote this endmarker or \#. We show that a message can be sent only at the beginning of period $\tau$, when head $A_{p}$ is still close to $\rho$. If any automaton sends a message during period $\tau$, then by properties of $b$, it happens when it scans a single $b$, i.e. within $O(|b|)$ steps. At this time, the head of $A_{p}$ makes $O(|b|)$ moves on the input. After receiving the first message we enter a new phase of the internal period, when a second message can be sent. Notice that again it must happen within $O(|b|)$ steps, if it happens at all. Again the distance of $A_{p}$ from $\rho$ may increase by at most $O(|b|)$. Since totally at most $c$ messages are sent during the whole computation, it follows that the distance of $A_{p}$ from $\rho$ is $O(c \cdot|b|)$ at the moments when a message is sent during internal period $\tau$.

At this point we are ready to prove Theorem 1 . Assume that $M$ is an $l$-automata system that recognizes $L_{k}^{l}$ with at most $k-1$ messages. Now we consider an input $x \# y_{1} \# \ldots \# y_{k}$ defined as follows. For each $i$, word $y_{i}$ has the form $b_{z_{i}}$, where $b$ is given by Border Lemma, $x=(1 b)^{n}$, for large enough $n,\left|y_{i}\right|>l \cdot|x|$, and $K\left(z_{i}\right) \geq\left|z_{i}\right|-c \cdot \log \left|z_{i}\right|$, for $i \leq k$. Let the symbols on positions $|x|, 2|x|, \ldots,(l-1)|x|$ in $y_{1}, y_{2}, \ldots, y_{k}$ be called important symbols. We assume that all important symbols are ones. Let $y_{i, j}$ be the subword of $y_{i}$ consisting of symbols on positions $j \cdot|x|-\lfloor\sqrt{n}\rfloor, \ldots, j \cdot|x|+\lfloor\sqrt{n}\rfloor$. 
Without loss of generality we assume that $M$ accepts at the moment when all heads stand at the first symbol of the input. By Border Lemma, if a message is sent, at least one head is at distance $O(|b|)$ from an \# or an endmarker. So at most $l-1$ automata have their heads near the important symbols. Hence there are $i, j$ such that no message is sent if a head is inside $y_{i, j}$. By Confusion Lemma, the substring $y_{i, j}$ may be replaced by a string $y_{i, j}^{\prime}$ having the same clock characterization, but a zero in the middle. Since no message is sent when a head is inside $y_{i, j}\left(y_{i, j}^{\prime}\right.$ respectively), the same clock characterizations guarantee the same result at the end of the computation (note that the heads are outside $y_{i, j}\left(y_{i, j}^{\prime}\right)$ at this moment). A contradiction, since the modified word is not in $L_{k}^{l}$

Theorem 1.

\section{Hierarchy between $\log \log n$ and $n$}

In this section we prove Theorems 2 and 4. The lower bound arguments are based on Lemma 4. It says that $\Omega(m)$ messages are needed in order to check whether the lengths

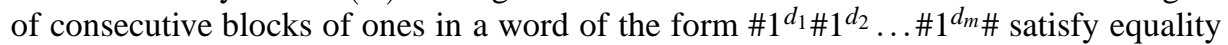
$d_{i}=\alpha\left(d_{i-1}, d_{i-2}\right)$ for $i=3, \ldots, m$ and a function $\alpha: \mathbb{N}^{2} \rightarrow \mathbb{N}$ defined as below.

Lemma 4. Let $\alpha: \mathbb{N}^{2} \rightarrow \mathbb{N}$ be a function such that for any $a_{1}>a_{2}$ and $b$ it holds that $\alpha\left(a_{1}, b\right) \geq a_{1}$ and $\alpha\left(a_{1}, b\right)>\alpha\left(a_{2}, b\right)$. Let $f: \mathbb{N} \rightarrow \mathbb{N}$. Let $L_{\alpha, f}$ be an infinite subset of

$\left\{x \star w \star y \mid x, w, y \in(\Sigma \backslash\{\star\})^{*}, w=1^{d_{1}} \# \ldots \# 1^{d_{m}}, m \in \mathbb{N}, d_{i}=\alpha\left(d_{i-1}, d_{i-2}\right)\right.$ for $\left.i=3 \ldots m\right\}$

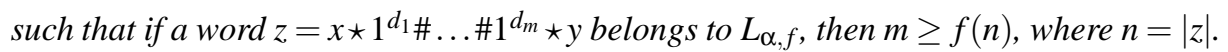
If $\alpha(x, y)=x$ for any $y>0$, then we assume additionally that $d_{1} \geq g(m)$ and $d_{2} \geq g(m)$ for some nondecreasing function $g: \mathbb{N} \rightarrow \mathbb{N}$ that is not bounded by a constant.

Then $L_{\alpha, f} \notin \operatorname{MESSAGE}(h(n))$, for any $h(n)=o(f(n))$.

Proof. Assume that there exists a multi-automata system $S$ that recognizes language $L_{\alpha, f}$ and uses $h(n)=o(f(n))=o(m)$ messages on $w$ for every input $x \star w \star y \in L_{\alpha, f}$.

First we shall show that no message is sent on a "long" sequence of "long" blocks of ones in the middle part of an input $z \in L_{\alpha, f}$. Then we shall find two blocks \#1 $1^{d_{i} \# \text { and }}$

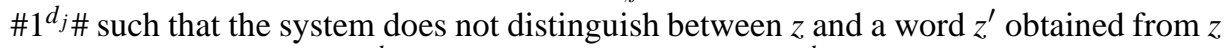

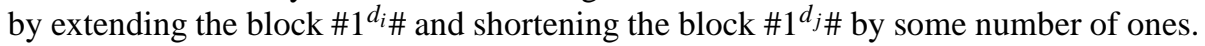

Let $Q_{S}$ be the set of states of the automata of $S$. We focus our attention on a set

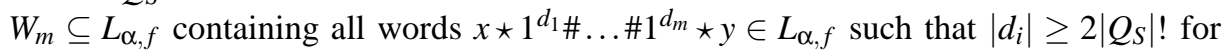
$i>m / 2$. We consider messages sent during computation of $S$ on a word $x \star w \star y \in W_{m}$. Let $w^{\prime}=\# 1^{d_{u}} \ldots \ldots 1^{d_{u+a-1}} \#$ be a subword of $w$ such that no message was sent while any automaton was reading $w^{\prime}$. Since $h(n)=o(n)$, we can choose $w^{\prime}$ so that $u>m / 2$ and $a$ is a large constant (to be determined later), if $m$ is large enough.

We inspect more closely behavior of $S$ on $w^{\prime}$. For each block of ones in $w^{\prime}$, we define a trace of computation on $w^{\prime}$ : for $u \leq i \leq u+a-1$, trace $T_{i}$ is the set of tuples

$$
\left(A_{j}, q, s, k, q_{f}, s_{f}, q_{t}, s_{t}\right)
$$

where $A_{j}$ is an automaton of $S, \quad q, q_{f}, q_{t} \in Q, \quad s, s_{f}, s_{t} \in\{$ Left, Right $\}$, satisfying the following properties: during a computation of $A_{j}$ on $w^{\prime}$ started at side $s$ of $w^{\prime}$ in state $q$, 
block $i$ of 1 's is entered for the $k$ th time on side $s_{f}$ in state $q_{f}$ and is left on side $s_{t}$ in state $q_{t}$. Observe that $k \leq\left|Q_{S}\right|$, so the number of possible traces is bounded by some function of $\left|Q_{S}\right|$. But $\left|Q_{S}\right|$ is a constant, so we may take a number $a$ bigger than the number of possible traces. Then there are two blocks of 1's in $w^{\prime}$, say the $i$ th and the $j$ th, with the same traces.

Let us look closer at computation of automata of $S$ on the $i$ th and the $j$ th blocks in $w^{\prime}$. Each automaton entering such a block either leaves it on the same side, or goes to the opposite end. In the first case, the automaton cannot reach more than $\left|Q_{S}\right|$ symbols inside the block, since otherwise it would loop and go to the opposite end. In the second case, after at most $\left|Q_{S}\right|$ steps the automaton enters a loop. The number of symbols read during the loop, say $r$, is at most $\left|Q_{S}\right|$. Observe that we may extend the block of ones by a multiple of $r$ and the automaton reaches the opposite end in the same state. We may also shorten it by a multiple of $r$ provided that we leave place for initial steps before going into the loop. In our construction we shorten or extend the blocks of ones by $\left|Q_{S}\right|$ ! ones. The trick is that this number is a multiple of any $r$ considered above. The second point is that the blocks have length at least $2\left|Q_{S}\right|$ !, so we leave place for automata to get into the loop or return to the side of arrival within the remaining part of the block.

In order to fool $S$, we replace $1^{d_{i}}$ by $1^{d_{i}+\left|Q_{S}\right| !}$ and $1^{d_{j}}$ by $1^{d_{j}-\left|Q_{S}\right| !}$. As already noticed, this leaves the traces unchanged. It follows that blocks $i$ and $j$ are traversed in the same way and the same number of times as before. The only difference is that the runs through block $i$ are longer than before, and the runs through block $j$ are shorter than before. However, the additional time lost on block $i$ is equal to the time saved on block $j$. This follows from the fact that each automaton goes through the same loops on both blocks and that we have extended and shortened them by the same number of ones. Since each automaton spends the same time on $w^{\prime}$ as before, $S$ cannot detect any change. However, the changed word does not satisfy the conditions from the definition of $L_{\alpha, f}$.

Now we prove the lower bound of Theorem 2. By Lemma 4, it suffices to show that the number of blocks of ones is $\Omega(\log \log n)$ for infinitely many words $x \in L_{a, b}$.

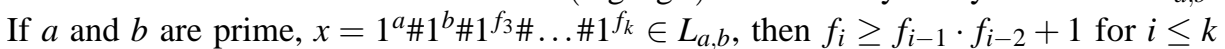
by Chinese Reminder Theorem. For the shortest word $x \in L_{a, b}$ with $k$ blocks of ones we have even $f_{i}=f_{i-1} \cdot f_{i-2}+1$. Then it is easy to check that $f_{k}=\Omega\left(a^{F_{k-3}} b^{F_{k-2}}\right)$ and $f_{k}=O\left(2^{k} a^{F_{k-3}} b^{F_{k-2}}\right)$ where $F_{i}$ is the $i$ th Fibonacci number. Furthermore $f_{k} \geq k+\sum_{i=1}^{k-1} f_{i}$ for large enough $k$. So, $f_{k} \leq n \leq 2 f_{k}$ and therefore $k=\Theta(\log \log n)$.

A two-automata system may recognize $L_{a, b}$ as follows:

1. The first automaton checks that the lengths of the first two blocks of ones are equal to $a$ and $b$.

2. For $i=3, \ldots, k$ the system checks that $f_{i}=1 \bmod f_{i-2}$ and $f_{i}=1 \bmod f_{i-1}$ (if not, then it rejects)

3. Accept

Checking that $f_{i}=1 \bmod f_{i-1}$ is performed as follows: automaton $A_{1}$ goes from the left to the right over block $i$ while automaton $A_{2}$ loops between the "ends" of block $i-1$. They finish when the automaton $A_{1}$ reaches symbol \# and sends a message. Since $A_{2}$ may remember in states if it has left the symbol \# in the previous step, the result may be easily established.

Now we sketch the proof of Theorem 4. Let $M$ be a 2-tape Turing machine which makes exactly $f(n)$ steps on input $1^{n}$. We construct a language $L_{M}$ which requires 
$\Theta\left(f^{2}(\log \log n)\right)$ messages. $L_{M}$ consists of words of the form $z_{k}=b_{k} \& c_{k} \& h_{k} \& l_{k} \star e_{k} \star$, where subwords $b_{k}, c_{k}, h_{k}$ are responsible for "computing" $f(k)$, and $l_{k}, e_{k}$ guarantee that $f^{2}(k)$ messages are necessary. Let $b_{k}$ be the word in $L_{2,3}$ consisting of $k$ blocks of ones and $c_{k}=1^{k}$. In this way we fix the input $1^{k}$ for $M$. (Word $b_{k}$ guarantees that the input length is double-exponential in the length of $c_{k}$ on which we simulate machine $M$.) Then $h_{k}=k_{1} \# k_{2} \# \ldots \# k_{f(k)}$, where $k_{i}$ is the contents of the tapes of $M$ (in consecutive configurations) with head positions marked with symbols denoting the state of $M$. Finally, $l_{k}=\left(1^{f(k)-1} \#\right)^{f(k)}$ and $e_{k}=\left(1^{f^{2}(k)} \#\right)^{f^{2}(k)}$.

It is easy to check that if $\left|z_{k}\right|=n$, then $k=\Omega(\log \log n)$. The bound $\Omega\left(f^{2}(\log \log n)\right)$ on the number of messages necessary to recognize $L_{M}$ follows from Lemma 4 , since we have to check that $f^{2}(k)$ blocks of ones in $e_{k}$ have the same length. It is tedious but relatively easy to show that $O\left(f^{2}(k)\right)$ messages suffice to check the whole structure of $z_{k}$.

Theorem 4

\section{Superlinear size of communication}

In this section we sketch the proof of Theorem 5. We assume that the first input matrix is listed row by row, the second matrix is listed column by column (rows and columns are separated by \# symbols). Moreover, the output matrix is printed out column by column. An implementation of the standard algorithm for multiplying matrices requires $O\left(n^{3 / 2}\right)$ messages. So the non-trivial part of this theorem is to show the lower bound claimed.

Consider an $l$-automata system $M$ computing product of matrices over $\mathbb{F}_{2}$. We count the write operations on the output tape as messages (this does not change the number of messages significantly). Consider a computation of $M$ on an input $x \# y$ describing two $N \times N$ matrices $X$ and $Y, n=2 N^{2}+2 N$, such that $K(x \# y) \geq 2 N^{2}-c \log 2 N^{2}$. We divide computation into phases during which the automata generate $s(N)$ bits of the output (number $s(N)$ will be chosen in a moment). We prove the following key property:

Claim A. Let functions s and $k$ satisfy $s(N)=\omega(\log N), s(N)=o(N / k(N))$. Then during computation on $x \# y$ at least $k(N)$ messages are sent during each phase. So totally, $N^{2}$. $k(N) / s(N)$ messages must be sent.

W.l.o.g. we may assume that $s(N)$ divides $N$. Then during each phase the output bits generated belong to the same column. Consider a phase $\mathcal{E}$ on input $x \# y$ and its output bits $c_{p, j}, \ldots, c_{p+s(N)-1, j}$ of column $c_{j}$. These bits are the scalar products of rows $w_{p}, \ldots, w_{p+s-1}$ of $X$ by column $y_{j}$ of $Y$. Assume that during phase $\mathcal{E}$ less than $k(N)$ messages are sent. We show that this contradicts the assumption that $K(x \# y) \geq 2 N^{2}-$ $c \log N^{2}$.

By our assumption, there is an interval $B$ in $y_{j}$ of length $b=N /(l \cdot k(N))$ such that no message is sent while any automaton scans $B$ during phase $\mathcal{E}$. Computation of $M$ on $B$ may be described by a clock characterization. For each automaton it contains tuples describing state of automaton while entering and leaving $B$ and the time spent on $B$. Since there are constant number of states at which an automaton may enter and leave $B$ and $O(\log n)$ bits suffice to describe computation time, each clock characterization has $O(\log n)$ bits. Obviously, the output bits generated during phase $\mathcal{E}$ are uniquely determined by input $x \# y$ without $B$, the initial configuration of $M$ at the beginning of phase $\mathcal{E}$, and clock characterization of $B$. All these data may be described by a string of 
length $2 N^{2}-b+O(l \cdot \log n)$. The next claim shows that we may uniquely describe $B$ by a relatively short string, given the output of $M$ during phase $\mathcal{E}$.

Claim B . There are at most $2^{b-s(N)}$ vectors $u$ of length $b$ such that replacing the contents of $B$ by $u$ inside input $x \# y$ does not change the output bits $c_{p, j}, c_{p+1, j}, \ldots, c_{p+s(N)-1, j}$.

Let $Z$ be the submatrix, consisting of those elements of rows $w_{p}, \ldots, w_{p+s-1}$ of $X$, which are multiplied by elements of $B$ while determining the output of phase $\mathcal{E}$ by the standard algorithm. Clearly, $Z$ is an $s(N) \times b$ matrix. We claim that the rows of $Z$ are linearly independent. Otherwise, we could represent a row of $Z$ as a linear combination of its remaining rows, say $Z_{k}=\sum_{i \neq k} v_{i} \cdot Z_{i}$. Then we could encode $Z$ by the vectors $Z_{1}, \ldots, Z_{k-1}, Z_{k+1}, \ldots, Z_{s(n)}$, number $k$ and the coefficients $v_{1}, \ldots, v_{s(n)}$. Such a representation would consist of $|Z|-b+s(N)+O(\log N)$ bits. This would lead to compression of the input $x \# y$ into $2 N^{2}-b+s(N)+O(\log N)$ bits, so below $2 N^{2}-c \log 2 N^{2}$.

Let $e$ denote the output bits of phase $\mathcal{E}$. Observe that given $e$, the rows $w_{p}, \ldots, w_{p+s-1}$, and the contents of $y_{j}$ except $B$, the product $Z \cdot B$ is uniquely determined. The number of solutions of equation $Z \cdot B=d$ is $2^{b-s(N)}$, since $Z$ contains $s(N)$ linearly independent rows and the number of (unknown) bits in $B$ equals $b$. Claim B

Input $x \# y$ can be encoded by: $x \# y$ without $B$ and \# symbols (together $2 N^{2}-b$ bits), the configuration of $M$ at the beginning of phase $\mathcal{E}$, the position of $B$, the clock characterization of $B$ (all of length $O(l \log n)$ ), and the index of the contents of $B$ in the lexicographic ordering among all strings $u$ that generate output bits $c_{p, j}, \ldots, c_{p+s(N)-1, j}$ (at most $b-s(N)$ bits, by Claim B ). The length of this encoding is $\left(2 N^{2}-b\right)+O(l$. $\log n)+(b-s(N))=2 N^{2}+O(l \cdot \log n)-s(N)$. Since $s(N)=\omega(\log N), \log n \approx 2 \log N$, and $l$ is a constant, this encoding is shorter than $2 N^{2}-c \log N^{2}$. Contradiction. Claim A Let $\beta(n)=\omega\left(\log ^{2} n\right), \gamma(n)=\beta(n) / \log ^{2}(n)$. We take $s(N)=\gamma^{1 / 3}(n) \log n$ and $k(N)=$ $N /\left(\gamma^{2 / 3}(n) \log N\right)$. These functions satisfy conditions of Claim A, so we get that $N^{3} / \beta(n)$ messages are necessary. This concludes the proof of Theorem 5.

\section{References}

1. P. Beame, M. Tompa, P. Yan, Communication-Space Tradeoffs for Unrestricted Protocols, SICOMP 23 (1994), 652-661.

2. A. Borodin, S. Cook, A Time-Space Tradeoff for Sorting on a General Sequential Model of Computation, SICOMP 11 (1982), 287-297.

3. A.O. Buda, Multiprocessor Automata, IPL 25 (1987), 157-161.

4. M. Dietzfelbinger, The Linear-Array Problem in Communication Complexity Resolved, in Proc. STOC'97, 373-382.

5. P. Duriš, Z. Galil, A Time-Space Tradeoff for Language Recognition, MST 17 (1984), 3-12.

6. P. Durišs, T. Jurdziński, M. Kutyłowski, K. Loryś, Power of Cooperation and Multihead Finite Systems, in Proc. ICALP'98, 896-907.

7. M. Karchmer, Two Time-Space Tradeoffs for Element Distinctness, TCS 47 (1986), 237-246.

8. E. Kushilevitz, N. Nisan, Communication Complexity, Cambridge University Press, 1997.

9. T. Lam, P. Tiwari, M. Tompa, Trade-offs between Communication and Space, JCSS 45 (1992), 296-315.

10. M. Li, P. Vitanyi, An Introduction to Kolmogorov Complexity and its Applications, SpringerVerlag, 1993.

11. Y. Yesha, Time-Space Tradeoffs for Matrix Multiplication and the Discrete Fourier Transform of Any General Sequential Random-Access Computer, JCSS 29 (1984) 183-197. 